\title{
Radioiodinated Small-Molecule Tyrosine Kinase Inhibitor for HER2-Selective SPECT Imaging
}

\author{
Longguang Tang*1,2, Chenyu Peng*1, Bowen Tang ${ }^{3}, \mathrm{Zijing} \mathrm{Li}^{1}$, Xiangyu Wang ${ }^{1}$, Jindian $\mathrm{Li}^{1}$, Fei Gao ${ }^{1}$, Lumei Huang ${ }^{1}$, \\ Duo $\mathrm{Xu}^{1}$, Pu Zhang ${ }^{1}$, Rongqiang Zhuang ${ }^{1}$, Xinhui $\mathrm{Su}^{4}$, Xiaoyuan $\mathrm{Chen}^{2}$, and Xianzhong Zhang ${ }^{1}$ \\ ${ }^{1}$ State Key Laboratory of Molecular Vaccinology and Molecular Diagnostics and Center for Molecular Imaging and Translational \\ Medicine, School of Public Health, Xiamen University, Xiamen, China, ${ }^{2}$ Laboratory of Molecular Imaging and Nanomedicine (LOMIN), \\ National Institute of Biomedical Imaging and Bioengineering (NIBIB), National Institutes of Health, Bethesda, Maryland; ${ }^{3}$ School of \\ Pharmaceutical Science, Xiamen University, Xiamen, China; and ${ }^{4}$ Zhongshan Hospital, affiliated with Xiamen University, Xiamen, China
}

One of the most clinically relevant molecular aberrations in breast cancer is overexpression of human epidermal growth factor receptor type 2 (HER2). We aimed to develop a radiolabeled tyrosine kinase inhibitor for HER2-targeted breast cancer imaging. In this study, a radioiodinated analog (125/131|-IBA-CP) of the HER2-selective inhibitor CP724,714 was prepared and evaluated in HER2-positive or -negative subcutaneous human breast cancer xenografts. Methods: The CP724,714 analog IBA-CP was synthesized and assayed for its inhibitory activities against HER2 and 6 other tyrosine kinases. ${ }^{125 / 131}$ I-IBA$\mathrm{CP}$ was prepared using a copper-mediated radioiodination method with enhanced labeling yield and molar activity. In vitro biologic activity, including specific and nonspecific binding of ${ }^{131}$ I-IBA-CP to its HER2 kinase target, was assessed in different cell lines. In vivo small-animal 125I-IBA-CP SPECT imaging and biodistribution studies were conducted on mice bearing HER2-positive, HER2-negative, or epidermal growth factor receptor (EGFR)-positive tumors. Nonradioactive IBA-CP and the EGFR inhibitor erlotinib were used as blocking agents to investigate the binding specificity and selectivity of $125 / 131$ I-IBA-CP toward HER2 in vitro and in vivo. Additionally, ${ }^{125 / 131}$ I-ICP was prepared by direct radioiodination of CP724,714 for comparison with ${ }^{125 / 131}$ |-IBACP. Results: IBA-CP displayed superior in vitro inhibitory activity (halfmaximal inhibitory concentration, $16 \mathrm{nM}$ ) and selectivity for HER2 over 6 other cancer-related tyrosine kinases. ${ }^{125 / 131}$ I-IBA-CP was prepared in a typical radiochemical yield of about $65 \%$ (decay-corrected), radiochemical purity of more than $98 \%$, and molar activity of $42 \mathrm{GBq} / \mathrm{\mu mol}$ at the end of synthesis. SPECT imaging revealed significantly higher uptake of ${ }^{125}$ I-IBA-CP than of ${ }^{125}$ I-ICP in the HER2-positive MDA-MB453 tumors. Uptake in the HER2-negative MCF-7 tumors was much lower. Binding of ${ }^{125}$ |-IBA-CP in the MDA-MB-453 tumors was blocked by coinjection with an excess amount of IBA-CP, but not by erlotinib. Conclusion: The radiolabeled HER2-selective inhibitor ${ }^{125 / 131}$ |-IBA-CP is a promising probe for in vivo detection of HER2-positive tumors.

Key Words: HER2; tyrosine kinase inhibitor; radioiodinated IBA-CP; breast cancer; small-animal SPECT

J Nucl Med 2018; 59:1386-1391

DOI: 10.2967/jnumed.117.205088

Received Nov. 9, 2017; revision accepted Mar. 23, 2018.

For correspondence or reprints contact: Xianzhong Zhang, Center for Molecular Imaging and Translational Medicine, School of Public Health, Xiamen University, Xiang'an South Rd., Xiang'an District, Xiamen 361102, China.

E-mail: zhangxzh@xmu.edu.cn

${ }^{*}$ Contributed equally to this work.

Published online Apr. 13, 2018.

COPYRIGHT (C) 2018 by the Society of Nuclear Medicine and Molecular Imaging.
$\mathbf{H}$ uman epidermal growth factor receptor type 2 (HER2) is a member of the epidermal growth factor receptor (EGFR) family (1), which is overexpressed in $20 \%-30 \%$ of breast cancers and in a similar subset of non-small cell lung cancers, gastric cancers, ovarian carcinomas, and colon carcinomas $(1,2)$. The HER2 expression status is routinely determined in tumor cells in vitro using an immunohistofluorescence or fluorescent in situ hybridization method $(3,4)$. However, the disease is usually verified by core-needle biopsy of an accessible lesion, a method that not only is invasive but also may not sample the largest tumor mass or other sites of disease $(5,6)$. Therefore, it is necessary to develop a more accurate and noninvasive method for the evaluation of HER2 expression in tumors throughout the body (7).

Radionuclide-based molecular imaging techniques such as PET and SPECT may represent an appropriate tool to obtain real-time, noninvasive, and quantitative information about HER2 expression in vivo with high sensitivity $(8-12)$. So far, several radiolabeled HER2-targeted imaging probes have been assessed, including antiHER2 antibodies (13-15) or their fragments (16), single-domain antibodies (17), diabodies (18), Affibody molecules (Affibody AB) $(19,20)$, and high-affinity peptide ligands $(21,22)$. Although most have shown high accumulation in tumor tissues, imaging with these large-molecule radiotracers has limitations due to the unfavorable receptor binding kinetics compared with their systemic clearance $(23,24)$. Furthermore, although protein- and peptide-based probes can image the extracellular tyrosine kinases, they cannot be used to assess the density and status of the intracellular kinases that are the primary targets of tyrosine kinase inhibitors (TKIs) (23). The smaller size and faster clearance of tailored TKI drugs provide a unique opportunity to examine the interaction between the drug and its HER2 target in vivo (25). However, the development of an optimum radiolabeled TKI imaging probe is challenging because of the great difficulty in balancing the lipophilicity to provide membrane permeability while also reducing nonspecific binding and hepatobiliary excretion (26). So far, no selective small-molecule inhibitor of HER2 has been reported as a nuclear imaging agent (14). Gniazdowska et al. synthesized ${ }^{99 \mathrm{~m}} \mathrm{Tc}$-labeled lapatinib in hopes of developing a potential SPECT imaging agent for HER2-positive breast cancer (27). However, because lapatinib is a dual HER2- and EGFR-targeting inhibitor, not a HER2-selective inhibitor, this kind of radiotracer will not be able to distinguish HER2 from EGFR in tumor cells.

To date, several compounds have been developed as HER2selective TKIs (28-31). One of them, CP724,714 (Fig. 1), was reported to be a potent inhibitor of HER2 receptor kinase (half-maximal 


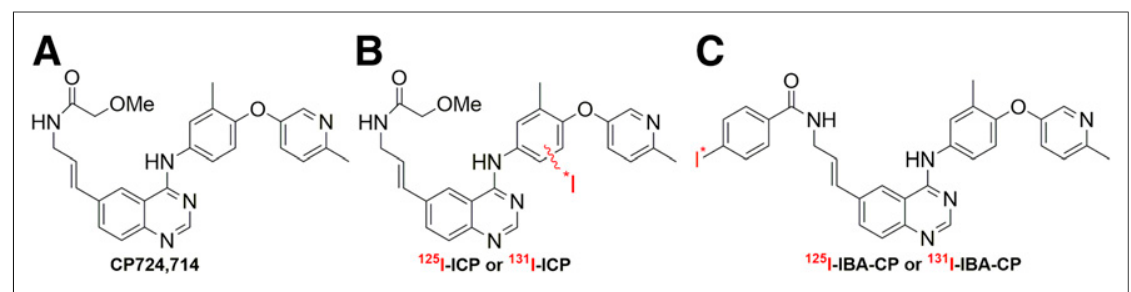

FIGURE 1. Chemical structures of CP724,714 (A), ${ }^{125}$ |- or ${ }^{131}$ I-labeled CP724,714 (B), and its analog (C).

inhibitory concentration $\left[\mathrm{IC}_{50}\right], 10 \mathrm{nM}$ ) in vitro, with 500 - to 1,000-fold selectivity over EGFR and other related receptor tyrosine kinases (32). Further, binding of CP724,714 to the HER2-active site was thought to tolerate structural modifications in its amide region (33). Hence, we prepared a novel analog of CP724,714 for radioiodination by replacing the 2-methoxyacetamide moiety with 4-iodobenzamide. Herein, we report the synthesis and evaluation of the radioiodinated analog, ${ }^{125}$ I-IBA-CP, as a potential SPECT imaging probe for in vivo detection of HER2 expression in breast cancer. The direct iodination product of CP724,714, ${ }^{125} \mathrm{I}-\mathrm{ICP}$, was also prepared for comparison.

\section{MATERIALS AND METHODS}

\section{Cell Culture and Animal Models}

Human breast cancer cell lines (BT-474, MDA-MB-453, MCF-7, and MDA-MB-468) were obtained from China Infrastructure of Cell Line Resources. The cells were cultured in RPMI 1640 medium (BT474, MCF-7, and MDA-MB-468) or in Leibovitz L15 medium (MDAMB-453) supplemented with $10 \%$ (v/v) heat-inactivated fetal bovine serum (Gibco) and penicillin/streptomycin (concentration of $100 \mathrm{U} / \mathrm{mL}$ for each). Cells were grown as a monolayer at $37^{\circ} \mathrm{C}$ in a humidified atmosphere containing $5 \% \mathrm{CO}_{2}$.

All animal studies were conducted in accordance with the guidelines of the Xiamen University Animal Care and Use Committee. Five- to 7-wk-old female BALB/C nude mice were injected subcutaneously into the right front flank with MDA-MB-453, MCF-7, or MDA-MB-468 cells $\left(1 \times 10^{7}\right.$ in $200 \mu \mathrm{L}$ of phosphate-buffered saline). The animals were used for in vivo studies when the tumors reached a size of $100-150 \mathrm{~mm}^{3}$ (2-3 wk after inoculation).

\section{Measurement of Lipophilicity (log P)}

The radiolabeled compound ( ${ }^{125} \mathrm{I}-\mathrm{IBA}-\mathrm{CP}$ or ${ }^{125} \mathrm{I}-\mathrm{ICP}, 0.74 \mathrm{MBq} / 0.1$ $\mathrm{mL}$ of 1-octanol each) was mixed with an equal volume of 1-octanol

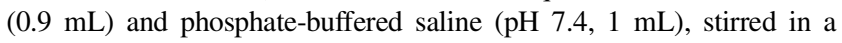
vortex mixer for $2 \mathrm{~min}$, and then centrifuged at 10,000 rpm for $2 \mathrm{~min}$ at room temperature to partition the layers. Aliquots of $0.1 \mathrm{~mL}$ each were taken from the organic and aqueous layers and counted in a well-type $\gamma$-counter. The distribution coefficient $P$ was calculated as the ratio of activity in the organic phase to that in the aqueous phase (as an average value from at least 3 independent measurements). The partition was done 3 times, and the $\log \mathrm{P}$ values were taken from the third partition.

\section{Preparation of Radiotracers}

The chemical structures for $\mathrm{CP} 724,714$ and its 2 radioiodinated analogs, ${ }^{125 / 131}$ I-IBA-CP and ${ }^{125 / 131}$ I-ICP, are shown in Figure 1 . The synthetic routes and methods are detailed in Supplemental Figure 1 (supplemental materials are available at http://jnm.snmjournals.org).

\section{In Vitro Studies}

The in vitro binding characteristics of ${ }^{131}$ I-IBA-CP were assessed using saturation and displacement cell-binding assays. The specificity of binding was also tested for cell lines with different levels of HER2 expression. For binding assays, ${ }^{131} \mathrm{I}-\mathrm{IBA}-\mathrm{CP}$ was prepared in high specific activity (42 MBq/nmol), and unlabeled IBA-CP was used as the competitor. Experiments were performed in triplicate according to a previously reported method (34). In brief, the day before the experiments, cells were seeded in 6-well plates at a concentration of $2.5 \times 10^{5}$ cells $/ \mathrm{mL}$ and then were incubated $\left(4^{\circ} \mathrm{C}, 2 \mathrm{~h}\right)$ with $43 \mathrm{nM}{ }^{131} \mathrm{I}-\mathrm{IBA}-\mathrm{CP}$ and increasing concentrations $(0.30 \mathrm{nM}-120 \mu \mathrm{M})$ of unlabeled IBA-CP.

\section{Small-Animal SPECT Imaging}

The experimental procedures with animals were approved by the Xiamen University Animal Care and Use Committee. SPECT/CT imaging was performed with a nanoScan SPECT/CT preclinical scanner (Mediso) on BALB/C nude mice bearing MDA-MB-453 or MCF-7 breast cancer xenografts. Imaging data were acquired at 1, 2, 3, and $4 \mathrm{~h}$ after the animals were intravenously injected with $18.5 \mathrm{MBq}$ of 125I-IBA-CP ( $n=4$ /group). Blocking studies were performed on MDAMB-453 mice ( $n=4 /$ group) at $3 \mathrm{~h}$ after coinjection of ${ }^{125}$ I-IBA-CP with IBA-CP $(200 \mu \mathrm{g} / \mathrm{mouse})$ or erlotinib $(200 \mu \mathrm{g} / \mathrm{mouse})$. SPECT imaging with ${ }^{125}$ I-ICP (18.5 MBq, intravenously) was also performed at 1 and $2 \mathrm{~h}$ after injection on mice with MDA-MB-453 xenografts ( $n=4$ /group). The duration of the SPECT/CT imaging sessions was about $30 \mathrm{~min}$ each.

\section{Biodistribution}

Biodistribution of ${ }^{131}$ I-IBA-CP in MDA-MB-453, MCF-7, or MDA-MB-468 tumor-bearing female mice was performed after intravenous injection of $0.18 \mathrm{MBq}$ of ${ }^{131} \mathrm{I}$-IBA-CP ( $n=4 /$ group). Blocking studies were performed on MDA-MB-453 tumor-bearing mice by coinjection with either IBA-CP (200 $\mu \mathrm{g} /$ mouse) or erlotinib (200 $\mu \mathrm{g} /$ mouse). A biodistribution study of ${ }^{131} \mathrm{I}$-ICP in MDA-MB-453 tumor-bearing mice was also conducted for comparison. The mice were killed and dissected $3 \mathrm{~h}$ after injection of the radioligand. Samples of tumor, blood, liver (without the gallbladder), and other major organs were collected and weighed, and the radioactivity in each sample was measured with a well-type $\gamma$-counter (2480 Wizard ${ }^{2}$; PerkinElmer). The results were expressed as percentage injected activity per gram of sample (\% ID/g, mean $\pm \mathrm{SD})$.

\section{Statistical Analysis}

Statistical analyses were performed using 1-way ANOVA followed by post hoc tests with SPSS statistical software (IBM), and the level of significance was set at a $P$ value of 0.05 .

\section{RESULTS}

\section{Chemistry and Radiochemistry}

Synthetic routes for the nonradioactive compound IBA-CP, its radiolabeling precursor, and others are shown in Supplemental Figure 1. All compounds were obtained in good yield and characterized by detailed spectroscopic analyses (Supplemental Figs. 2 and 3).

At first, radioiodinated IBA-CP was synthesized by a 2-step method (Supplemental Fig. 1A). To reduce the radiosynthesis time, an optimized 1-step radioiodination approach was developed to generate ${ }^{125} \mathrm{I}-\mathrm{IBA}-\mathrm{CP}$ or ${ }^{131} \mathrm{I}$-IBA-CP (Supplemental Fig. 1B) with a radiochemical yield of $65.3 \% \pm 5.2 \%(n=6)$ as measured by high-performance liquid chromatography. After purification by high-performance liquid chromatography, ${ }^{125}$ I-IBA-CP was obtained in more than $98 \%$ radiochemical purity (Supplemental Fig. 4) and a molar activity of about $42 \mathrm{GBq} / \mu \mathrm{mol}$. The total radiosynthesis time, including high-performance liquid chromatography purification, was $80-100 \mathrm{~min}$. 
CP724,714 was directly labeled using the standard IODOGEN method to prepare ${ }^{125} \mathrm{I}-\mathrm{ICP}$ or ${ }^{131} \mathrm{I}$-ICP (Supplemental Fig. 1C) in a high radiochemical yield of $90.3 \% \pm 5.2 \%(n=6)$ and a radiochemical purity of more than $95 \%$ (Supplemental Fig. 4E).

The $\log \mathrm{P}$ values of ${ }^{125} \mathrm{I}$-IBA-CP and ${ }^{125} \mathrm{I}$-ICP were $1.82 \pm 0.24$ $(n=3)$ and $2.71 \pm 0.21(n=3)$, respectively (Supplemental Table 1$)$, indicating that ${ }^{125} \mathrm{I}-\mathrm{IBA}-\mathrm{CP}$ is less lipophilic than ${ }^{125} \mathrm{I}-\mathrm{ICP}$.

\section{In Vitro and In Vivo Stability Study}

${ }^{125}$ I-IBA-CP was stable in both normal saline and murine plasma at $37^{\circ} \mathrm{C}$, with more than $96 \%$ of the parent compound intact after $4 \mathrm{~h}$ of incubation (Supplemental Fig. 5). The in vivo metabolic stability of ${ }^{131}$ I-IBA-CP and ${ }^{131} \mathrm{I}$-ICP was measured by analyzing the radiometabolites in the liver, blood, and urine at different postinjection times. Results from these experiments indicated that ${ }^{131} \mathrm{I}$ IBA-CP was much more stable than ${ }^{131}$ I-ICP in vivo (Supplemental Figs. 6 and 7). Measurement of thyroid activity uptake in normal mice confirmed that in vivo deiodination of ${ }^{131}$ I-IBA-CP was much less than that of ${ }^{131} \mathrm{I}$-ICP (Supplemental Fig. 8). As illustrated in our previous work $(35,36)$, the radioiodinated compounds obtained by the copper-mediated method showed higher in vitro and in vivo stability than those obtained by traditional electrophilic labeling methods using oxidizing agents such as IODO-GEN. This higher stability may be due to the fact that the copper-mediated method places the iodine in an electron-deficient aromatic ring whereas direct labeling introduces the iodine into an electron-rich one.

\section{In Vitro Inhibition of HER2 Kinase and Selectivity Assays}

CP724,714 and its analog IBA-CP were evaluated as inhibitors of HER2 and EGFR kinases in an assay of poly-Glu-Tyr phosphorylation by recombinant intracellular domains of the respective kinases, as previously described (30). Under the experimental conditions, CP724,714 inhibited the activity of HER2 with an $\mathrm{IC}_{50}$ value of $11.3 \mathrm{nM}$ (Supplemental Table 2), consistent with a previously reported result $\left(\mathrm{IC}_{50}, 10 \mathrm{nM}\right)$ (32). Similarly, the analog IBA-CP selectively inhibited the kinase activity of HER2 ( $\mathrm{IC}_{50}$, $16.5 \mathrm{nM})$ and was 100 -fold less potent $\left(\mathrm{IC}_{50}, 2,340 \mathrm{nM}\right)$ as an inhibitor of EGFR tyrosine kinase (Supplemental Tables 2 and 3). To assess its kinase selectivity, IBA-CP was further evaluated as an inhibitor of 5 other cancer-related kinases (HER4, FGFR-1, PDGFR $\beta$, VEGFR-2, and c-Met) and found to be more than 1,000-fold less potent than for HER2 (Supplemental Table 3).

\section{Cellular Binding Specificity and Cell Internalization}

The HER2 and EGFR protein levels in different breast carcinoma cells were evaluated by Western blot (Fig. 2A). HER2 protein was overexpressed in MDA-MB-453 and BT-474 cells, whereas EGFR protein was overexpressed in MDA-MB-468 cells. More quantitative measurements of HER2 and EGFR expression came from flow cytometry analysis in 4 different cell lines, showing results similar to those from Western blot analysis (Supplemental Figs. 9 and 10). Competition studies revealed that ${ }^{131}$ I-IBA-CP binding was displaced by increasing amounts of the unlabeled compound IBA$\mathrm{CP}$, with the $\mathrm{IC}_{50}$ value calculated at $19 \mathrm{nM}$ (Fig. 2C). Saturation analysis displayed a single class of high-affinity binding sites with a mean equilibrium dissociation constant of $18 \mathrm{nM}$ for ${ }^{131}$ I-IBA-CP in the MDA-MB-453 cell line (Fig. 2D). Binding specificity was confirmed using cell lines with different levels of HER2 expression, with ${ }^{131}$ I-IBA-CP cell uptake values correlating positively with HER2 expression levels. Binding to HER2-negative cells was low and almost the same as that in the blocking groups, in which a 100 -fold excess of nonradioactive compound IBA-CP was added to block the binding of ${ }^{131}$ I-IBA-CP to HER2 receptors (Fig. 2B).

In addition, we performed a Western blot analysis to determine the levels of
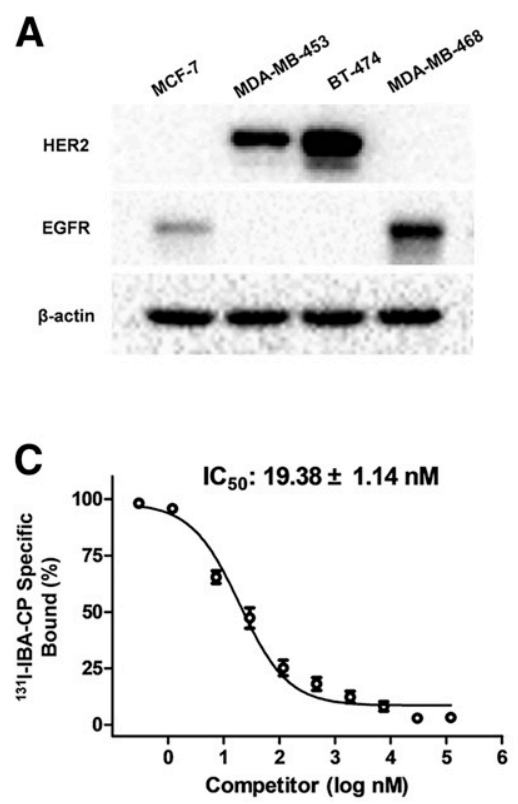
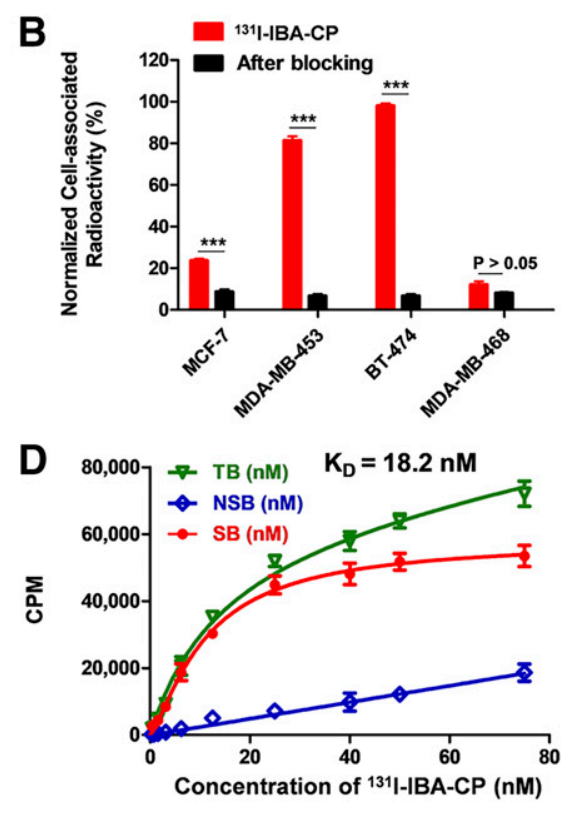

FIGURE 2. (A) HER2 and EGFR protein levels in different cells evaluated by Western blot. (B) Binding of ${ }^{131}$ I-IBA-CP to cells with different levels of HER2 expression (cell line vs. normalized cell-associated radioactivity and effect of preincubation with IBA-CP on binding. Data are mean \pm $\mathrm{SD}(n=3)$. (C) Representative results of competition-binding assay using MDA-MB-453 cells. (D) Representative results of saturation assay using MDA-MB-453 cells. NSB = nonspecific binding; $\mathrm{SB}=$ specific binding; $\mathrm{TB}=$ total binding. ${ }^{\star \star \star} P<0.001$. HER2 and HER-2 tyrosine phosphorylation, EGFR, and EGFR tyrosine phosphorylation proteins before and after incubation with different concentrations of IBA-CP or CP724,714 to further confirm their HER2specific inhibitory activity in BT-474 and MD-MB-468 cell lines (Supplemental Fig. 11).

Cell internalization assays were conducted with ${ }^{131}$ I-IBA-CP and ${ }^{131}$ I-ICP to compare their intracellular accumulation in MDA-MB-453 cells. Slightly more ${ }^{131} \mathrm{I}-\mathrm{ICP}$ was internalized than ${ }^{131} \mathrm{I}-\mathrm{IBA}-\mathrm{CP}$ (Supplemental Fig. 12), probably because of the less lipophilic nature of IBA-CP (Supplemental Table 1).

\section{SPECT/CT Imaging with ${ }^{125}$ I-IBA-CP and ${ }^{125} \mathrm{I}-\mathrm{ICP}$}

Given the favorable tumor cell retention and HER2 specificity of ${ }^{125}$ I-IBA-CP in vitro, small-animal SPECT/CT imaging was performed on nude mice with subcutaneous MDA-MB-453 and MCF-7 tumors. ${ }^{125} \mathrm{I}-\mathrm{IBA}-\mathrm{CP}$ was shown to have high liver uptake and to clear through both renal and hepatobiliary routes, with tumor accumulation in the HER2-positive group 
peaking at $3 \mathrm{~h}$ after injection (Fig. 3A). Uptake levels of ${ }^{125}$ I-IBA$\mathrm{CP}$ in other organs were similar in both groups (Figs. 3A and $3 \mathrm{~B}$ ). The peak tumor-to-muscle activity ratio was significantly higher in MDA-MB-453 tumors (3.9, Fig. 3C) than in MCF-7 tumors (1.3, Fig. 3D) at $3 \mathrm{~h}$ after injection. For ${ }^{125} \mathrm{I}-\mathrm{ICP}$, higher uptake was seen in the liver region, with much less uptake in the tumor (Supplemental Fig. 13A), resulting in a peak tumor-to-muscle activity ratio of 2.3 at $1 \mathrm{~h}$ after injection (Supplemental Fig. 13B).

After imaging, the tumors were excised for analysis of HER2 expression by immunohistofluorescence. An excellent correlation was found between SPECT signal intensity and histopathologic measurement (Supplemental Fig. 14)

\section{Blocking Studies with IBA-CP and Erlotinib}

The binding specificity and selectivity of ${ }^{125}$ I-IBA-CP in MDAMB-453 tumors were evaluated by blocking experiments with IBA-CP and erlotinib. Coinjection of ${ }^{125}$ I-IBA-CP with nonradioactive IBA-CP significantly reduced uptake of radioactivity in the tumors (Fig. 4A and 4B). This blocking effect was further confirmed by immunohistochemistry analysis of available HER2 and phosphorylated HER2, shown in Supplemental Figure 15. On the other hand, coinjection with the EGFR inhibitor erlotinib had no blocking effect on ${ }^{125} \mathrm{I}$-IBA-CP uptake in MDA-MB-453 tumors. Taken together, these results demonstrate the binding specificity and selectivity of ${ }^{125}$ I-IBA-CP toward HER2.

\section{Biodistribution}

Biodistribution of ${ }^{131} \mathrm{I}-\mathrm{IBA}-\mathrm{CP}$ was studied in mice bearing MDA-MB-453, MCF-7, and MDA-MB-468 (HER2-negative, EGFR-positive) tumors, as well as in the MDA-MB-453 tumor group, with coinjection of IBA-CP or erlotinib as a blocking agent (Fig. 5). At $3 \mathrm{~h}$ after ${ }^{131} \mathrm{I}-\mathrm{IBA}-\mathrm{CP}$ injection, activity distribution patterns in normal organs were similar among the groups, with the highest uptake being in the liver, intestine, and kidneys (Fig. 5A). However, uptake of ${ }^{131}$ I-IBA-CP was remarkably higher in MDA-MB-453 tumors than in MCF-7 or MDA-MB-468 tumors

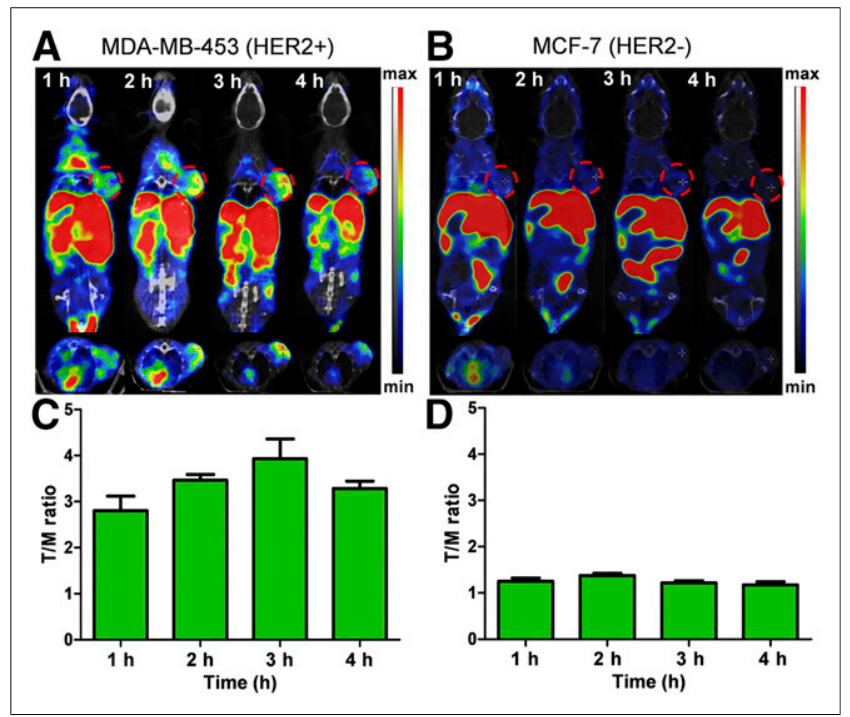

FIGURE 3. (A and B) Representative SPECT/CT images obtained 1, 2, 3 , and $4 \mathrm{~h}$ after injection of ${ }^{125}$ I-IBA-CP in mice bearing MDA-MB-453 (A) and MCF-7 (B) tumors (circled). (C and D) Respective tumor-to-muscle ratios obtained from images in $\mathrm{A}$ and $\mathrm{B}$. $n=4$; error bars correspond to SD.

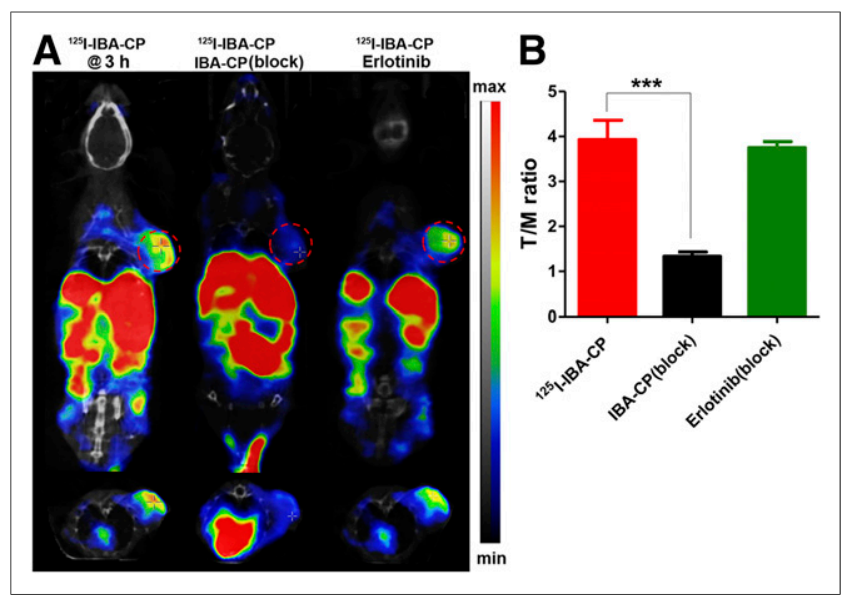

FIGURE 4. (A) Representative SPECT/CT images obtained $3 \mathrm{~h}$ after injection of ${ }^{125}$ I-IBA-CP in mice bearing MDA-MB-453 tumors (circled) with or without blocking dose of nonradioactive compound IBA-CP or erlotinib. (B) Quantification of SPECT imaging results. Data are mean \pm $\mathrm{SD}(n=4)$. T/M $=$ tumor-to-muscle ratio. ${ }^{* \star} P<0.001$.

$(1.05 \pm 0.25$ vs. $0.32 \pm 0.13$ and $0.38 \pm 0.05 \% \mathrm{ID} / \mathrm{g}, P<0.001)$ (Fig. 5B). Similarly, the tumor-to-muscle activity ratio was significantly higher in the HER2-positive group than in the 2 HER2-negative groups $(6.0 \pm 1.2$ vs. $1.9 \pm 0.7$ and $1.3 \pm 0.2, P<0.001)$ (Fig. 5 C).

The in vivo binding specificity and selectivity of ${ }^{131}$ I-IBA-CP was further evaluated in 2 independent experiments. One group of mice $(n=4)$ bearing HER2-positive MDA-MB-453 tumors was sacrificed and examined at $3 \mathrm{~h}$ after coinjection of an excess dose of nonradioactive IBA-CP with ${ }^{131}$ I-IBA-CP. As expected, coinjection dramatically reduced tumor uptake (from $1.05 \pm 0.25$ to $0.19 \pm 0.04 \% \mathrm{ID} / \mathrm{g}, P<0.001)$, as well as tumor-to-muscle activity ratio (from $6.0 \pm 1.2$ to $1.3 \pm 0.1$ ) (Fig. 5), whereas no significant changes in uptake were found in blood or any other organs (Fig. 5A). In another independent group, we tested whether coinjection with erlotinib interfered with the binding of ${ }^{131}$ I-IBACP. As seen in Figure 5, tumor uptake and tumor-to-muscle activity ratio were almost the same as in the control group, indicating that erlotinib did not compete with binding of the radiotracer. Hence, results from biodistribution studies further supported the in vivo binding specificity and selectivity of ${ }^{131}$ I-IBA-CP toward HER2.

Biodistribution of ${ }^{131} \mathrm{I}-\mathrm{ICP}$ at $3 \mathrm{~h}$ after injection was also studied in mice bearing MDA-MB-453 tumors and compared with that of ${ }^{131}$ I-IBA-CP. As shown in Supplemental Figure 16, ${ }^{131}$ I-ICP had much higher accumulation in the thyroid but less uptake in the tumor than ${ }^{131} \mathrm{I}-\mathrm{IBA}-\mathrm{CP}$.

\section{Molecular Modeling Studies}

Docking of CP724,714 and IBA-CP to the HER2 receptor and EGFR (Supplemental Fig. 17) showed that IBA-CP provided more negative free energy of binding to HER2 than to EGFR, at -10.28 and $-8.74 \mathrm{kcal} / \mathrm{mol}$, respectively. Significant differences in amino acid residues and noncovalent bonding were found between docking of IBA-CP to HER2 and docking of IBA-CP to EGFR. For the HER2/IBA-CP system, there were 3 hydrogen bonds in the hinge region. There was also a halogen bond between the iodine atom of IBA-CP and the side chain of LYS753, and an aromatic hydrogen bond between the toluene fragment and the side chain of ASP863. However, there was no hinge region, or interaction, for the EGFR/ IBA-CP complex. By comparison, CP724,714 displayed interactions 


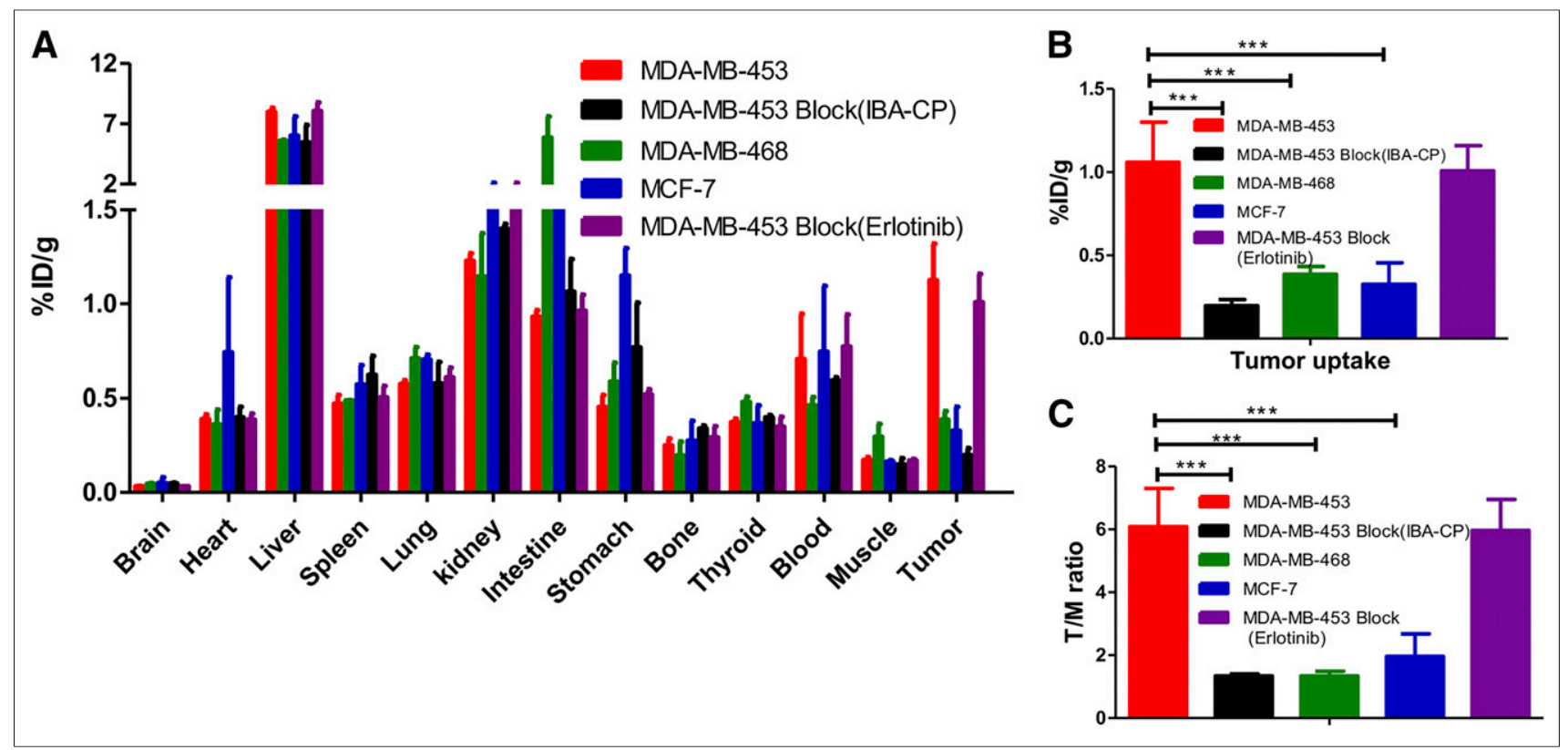

FIGURE 5. (A) Biodistribution of ${ }^{131}$ I-IBA-CP in MDA-MB-453 (HER2-positive), MDA-MB-468 (HER2-negative, EGFR-positive), and MCF-7 (HER2negative) tumor-bearing mice $3 \mathrm{~h}$ after injection and in MDA-MB-453 tumor-bearing mice coinjected with nonradioactive compound IBA-CP or erlotinib as blocking agent $30 \mathrm{~min}$ after injection. (B) ${ }^{131} \mathrm{I}$-IBA-CP tumor uptake from A. (C) ${ }^{131} \mathrm{I}-\mathrm{IBA}-\mathrm{CP}$ tumor-to-muscle ratio from A. Data are mean $\pm \mathrm{SD}(n=4)$. T/M = tumor-to-muscle ratio. ${ }^{\star \star \star} P<0.001$.

with HER2 and EGFR similar to those of IBA-CP, with free energies of binding at -10.56 and $-6.17 \mathrm{kcal} / \mathrm{mol}$, respectively. Collectively, the structural modification of CP724,714 to IBA-CP did not influence its affinity or specificity toward the tyrosine kinase domain of HER2.

\section{DISCUSSION}

In this article, we demonstrated the feasibility of labeling the HER2-selective inhibitor CP724,714 and its analog, IBA-CP, as radiotracers. Two radioiodinated tracers, ${ }^{125 / 131} \mathrm{I}-\mathrm{IBA}-\mathrm{CP}$ and ${ }^{125 / 131} \mathrm{I}-\mathrm{ICP}$, were thus prepared for evaluation of their in vitro and in vivo binding characteristics and in vivo imaging properties. To the best of our knowledge, this was the first study to show that radiolabeled small-molecule TKIs have the ability to image HER2 expression levels in vivo.

To reduce the radiosynthesis time, we labeled ${ }^{125} \mathrm{I}$-IBA-CP or ${ }^{131}$ I-IBA-CP by a copper-mediated radioiodination method using $\mathrm{CuCl} / 1,10-$ phenanthroline as a catalyst, which attached the radioiodine to an unactivated (nonphenolic) aromatic ring with high radiolabeling yield and molar activity (36). ${ }^{125}$ I-IBA-CP exhibited excellent stability both in vitro and in vivo. In vitro biologic activity assays indicated that, similar to CP724,714, IBA-CP was a HER2-selective TKI with negligible activity toward EGFR. As a small-molecule HER2-selective TKI, ${ }^{125}$ I-IBA-CP may have the ability to penetrate the cell membrane and thus can be used to better identify the status of the intracellular HER2 kinase (23). The easy radiolabeling protocol and favorable stability of ${ }^{125} \mathrm{I}-$ IBA-CP, coupled with its good binding affinity and high selectivity for HER2, encouraged us to further investigate its potential for in vivo tumor imaging with SPECT.

SPECT/CT imaging and biodistribution studies with radioiodinated IBA-CP in mice revealed rapid and comparatively high uptake in the subcutaneous HER2-positive MDA-MB-453 tumors and extremely low uptake in HER2-negative tumors (MDA-MB-
468 and MCF-7). MDA-MB-468 cells have a high level of EGFR expression; hence, the remarkably low uptake of ${ }^{131}$ I-IBA-CP indicated high selectivity of this radiotracer for HER2 over EGFR. The in vivo imaging results well matched the results of the immunohistofluorescence and immunohistochemistry analyses. Additionally, uptake of ${ }^{125}$ I-IBA-CP in HER2-positive tumors was effectively blocked by IBA-CP but not by the EGFR inhibitor erlotinib, further demonstrating that the binding of ${ }^{125} \mathrm{I}-\mathrm{IBA}-\mathrm{CP}$ in vivo was receptor-mediated, specific, and selective for HER2.

In comparison, ${ }^{125} \mathrm{I}-\mathrm{ICP}$, prepared from direct radioiodination of CP724,714, displayed lower uptake than ${ }^{125}$ I-IBA-CP in HER2positive tumors but higher accumulation in the liver region, as may be partly due to the higher lipophilicity of ${ }^{125} \mathrm{I}-\mathrm{ICP}(\log \mathrm{P}$ value of 2.71 vs. 1.82 for ${ }^{125}$ I-IBA-CP). Furthermore, it has been reported that CP724,714 can inhibit the hepatic efflux transporters, thus contributing to its hepatic accumulation and bile constituents (37). That may be the main reason for the higher liver uptake of ${ }^{125} \mathrm{I}$-ICP, which was also consistent with the high liver uptake found in imaging studies with other TKIs (38-40).

\section{CONCLUSION}

Our results demonstrate that the radioiodinated IBA-CP is HER2selective and thus may be used to assess HER2 expression in vivo by SPECT/CT imaging. After validation in humans, applications of this novel imaging agent may include patient stratification via assessment of HER2 kinase expression levels, as well as dose optimization and therapeutic effect monitoring through measurement of HER2 kinase inhibition and occupancy. Radionuclidebased imaging with a readily scalable synthetic small-molecule HER2 inhibitor is advantageous for clinical applications because of the high affinity to the target receptor and fast clearance from blood and normal organs. Nonetheless, the current imaging probe, although promising, displays only moderately specific binding signals. 
Further structure-activity optimization is needed to provide a radiotracer with improved in vivo performance, such as enhanced tumor uptake and reduced liver uptake.

\section{DISCLOSURE}

This study was financially supported by the National Key Basic Research Program of China (2014CB744503), the National Natural Science Foundation of China (21271030 and 81471707), and the Intramural Research Program, National Institute of Biomedical Imaging and Bioengineering, National Institutes of Health (ZIA EB000073). No other potential conflict of interest relevant to this article was reported.

\section{REFERENCES}

1. Yarden Y, Pines G. The ERBB network: at last, cancer therapy meets systems biology. Nat Rev Cancer. 2012;12:553-563.

2. Janjigian YY, Sanchez-Vega F, Jonsson P, et al. Genetic predictors of response to systemic therapy in esophagogastric cancer. Cancer Discov. 2018;8:49-58.

3. Sauter G, Lee J, Bartlett JM, Slamon DJ, Press MF. Guidelines for human epidermal growth factor receptor 2 testing: biologic and methodologic considerations. J Clin Oncol. 2009;27:1323-1333.

4. Wolff AC, Hammond MEH, Hicks DG, et al. Recommendations for human epidermal growth factor receptor 2 testing in breast cancer: American Society of Clinical Oncology/College of American Pathologists clinical practice guideline update. J Clin Oncol. 2013;31:3997-4013.

5. Scartozzi M, Bearzi I, Berardi R, Mandolesi A, Fabris G, Cascinu S. Epidermal growth factor receptor (EGFR) status in primary colorectal tumors does not correlate with EGFR expression in related metastatic sites: implications for treatment with EGFR-targeted monoclonal antibodies. J Clin Oncol. 2004;22:4772-4778.

6. Zidan J, Dashkovsky I, Stayerman C, Basher W, Cozacov C, Hadary A. Comparison of HER-2 overexpression in primary breast cancer and metastatic sites and its effect on biological targeting therapy of metastatic disease. Br J Cancer. 2005;93:552-556.

7. Capala J, Bouchelouche K. Molecular imaging of HER2-positive breast cancer: a step toward an individualized 'image and treat' strategy. Curr Opin Oncol. 2010;22:559-566.

8. Gebhart G, Flamen P, De Vries EGE, Jhaveri K, Wimana Z. Imaging diagnostic and therapeutic targets: human epidermal growth factor receptor 2. J Nucl Med. 2016;57(suppl):81S-88S.

9. Pereira PMR, Abma L, Henry KE, Lewis JS. Imaging of human epidermal growth factor receptors for patient selection and response monitoring: from PET imaging and beyond. Cancer Lett. 2018;419:139-151.

10. Henry KE, Ulaner GA, Lewis JS. Human epidermal growth factor receptor 2targeted PET/single-photon emission computed tomography imaging of breast cancer: noninvasive measurement of a biomarker integral to tumor treatment and prognosis. PET Clin. 2017;12:269-288.

11. Ulaner GA, Hyman DM, Lyashchenko SK, Lewis JS, Carrasquillo JA. ${ }^{89} \mathrm{Zr}$ trastuzumab PET/CT for detection of human epidermal growth factor receptor 2-positive metastases in patients with human epidermal growth factor receptor 2-negative primary breast cancer. Clin Nucl Med. 2017;42:912-917.

12. Ulaner GA, Lyashchenko SK, Riedl C, et al. First-in-human HER2-targeted imaging using ${ }^{89} \mathrm{Zr}$-pertuzumab PET/CT: dosimetry and clinical application in patients with breast cancer. J Nucl Med. November 16, 2017 [Epub ahead of print].

13. Cai W, Niu G, Chen X. Multimodality imaging of the HER-kinase axis in cancer. Eur J Nucl Med Mol Imaging. 2008;35:186-208.

14. Corcoran EB, Hanson RN. Imaging EGFR and HER2 by PET and SPECT: a review. Med Res Rev. 2014;34:596-643.

15. Mortimer JE, Bading JR, Colcher DM, et al. Functional imaging of human epidermal growth factor receptor 2-positive metastatic breast cancer using ${ }^{64} \mathrm{Cu}$-DOTA-trastuzumab PET. J Nucl Med. 2014;55:23-29.

16. Mendler CT, Gehring T, Wester H-J, Schwaiger M, Skerra A. ${ }^{89} \mathrm{Zr}$-labeled versus ${ }^{124}$ I-labeled $\alpha$ HER2 Fab with optimized plasma half-life for high-contrast tumor imaging in vivo. J Nucl Med. 2015;56:1112-1118.

17. Pruszynski M, Koumarianou E, Vaidyanathan G, et al. Improved tumor targeting of anti-HER2 nanobody through $\mathrm{N}$-succinimidyl 4-guanidinomethyl-3-iodobenzoate radiolabeling. J Nucl Med. 2014;55:650-656.
18. Reddy S, Shaller CC, Doss M, et al. Evaluation of the anti-HER2 C6.5 diabody as a PET radiotracer to monitor HER2 status and predict response to trastuzumab treatment. Clin Cancer Res. 2011;17:1509-1520.

19. Baum RP, Prasad V, Muller D, et al. Molecular imaging of HER2-expressing malignant tumors in breast cancer patients using synthetic ${ }^{111} \mathrm{In}$ - or ${ }^{68} \mathrm{Ga}$-labeled affibody molecules. J Nucl Med. 2010;51:892-897.

20. Niu G, Cai W, Chen X. Molecular imaging of human epidermal growth factor receptor 2 (HER-2) expression. Front Biosci. 2008;13:790-805.

21. Lee S, Xie J, Chen X. Peptides and peptide hormones for molecular imaging and disease diagnosis. Chem Rev. 2010;110:3087-3111.

22. Li L, Wu Y, Wang Z, et al. SPECT/CT imaging of the novel HER2-targeted peptide probe ${ }^{99 \mathrm{~m}} \mathrm{Tc}-\mathrm{HYNIC}-\mathrm{H} 6 \mathrm{~F}$ in breast cancer mouse models. J Nucl Med. 2017;58:821-826.

23. Tolmachev V, Stone-Elander S, Orlova A. Radiolabelled receptor-tyrosine-kinase targeting drugs for patient stratification and monitoring of therapy response: prospects and pitfalls. Lancet Oncol. 2010;11:992-1000.

24. Goldstein R, Sosabowski J, Vigor K, Chester K, Meyer T. Developments in single photon emission computed tomography and PET-based HER2 molecular imaging for breast cancer. Expert Rev Anticancer Ther. 2013;13:359-373.

25. Memon AA, Jakobsen S, Dagnaes-Hansen F, Sorensen BS, Keiding S, Nexo E. Positron emission tomography (PET) imaging with ${ }^{11} \mathrm{C}$-labeled erlotinib: a micro-PET study on mice with lung tumor xenografts. Cancer Res. 2009;69:873878.

26. Gelovani JG. Molecular imaging of epidermal growth factor receptor expressionactivity at the kinase level in tumors with positron emission tomography. Cancer Metastasis Rev. 2008;27:645-653.

27. Gniazdowska E, Koźmiński P, Bańkowski K, Łuniewski W, Królicki L. Synthesis, physicochemical and biological evaluation of technetium-99m labeled lapatinib as a novel potential tumor imaging agent of Her-2 positive breast cancer. Eur J Med Chem. 2014;87:493-499.

28. Nagasawa J, Mizokami A, Koshida K, Yoshida S, Naito K, Namiki M. Novel HER2 selective tyrosine kinase inhibitor, TAK-165, inhibits bladder, kidney and androgen-independent prostate cancer in vitro and in vivo. Int J Urol. 2006; 13:587-592.

29. Zhou Y, Brattain MG. Synergy of epidermal growth factor receptor kinase inhibitor AG1478 and ErbB2 kinase inhibitor AG879 in human colon carcinoma cells is associated with induction of apoptosis. Cancer Res. 2005;65:5848-5856.

30. Barbacci EG, Pustilnik LR, Rossi AM, et al. The biological and biochemical effects of CP-654577, a selective erbB2 kinase inhibitor, on human breast cancer cells. Cancer Res. 2003;63:4450-4459.

31. Jani JP, Barbacci G, Bhattacharya S, et al. CP-724714, a novel erbB2 receptor tyrosine kinase inhibitor for cancer therapy [abstract]. Cancer Res. 2004;64: 4637.

32. Jani JP, Finn RS, Campbell M, et al. Discovery and pharmacologic characterization of CP-724,714, a selective ErbB2 tyrosine kinase inhibitor. Cancer Res. 2007;67:9887-9893

33. Kath JC, Bhattacharya SK, Morris J, inventors; Pfizer Products Inc., assignee. Substituted bicyclic derivatives for the treatment of abnormal cell growth. WIPO patent WO/2001/098277A2. December 27, 2001.

34. Kramer-Marek G, Kiesewetter DO, Martiniova L, Jagoda E, Lee SB, Capala J. $\left[{ }^{18}\right.$ F $]$ FBEM-Z(HER2:342)-affibody molecule: a new molecular tracer for in vivo monitoring of HER2 expression by positron emission tomography. Eur J Nucl Med Mol Imaging. 2008;35:1008-1018.

35. Zhang P, Zhuang R, Guo Z, Su X, Chen X, Zhang X. A highly efficient coppermediated radioiodination approach using aryl boronic acids. Chemistry. 2016;22: 16783-16786.

36. Zhang $\mathrm{P}$, Zhuang $\mathrm{R}$, Wang $\mathrm{X}$, et al. Highly efficient and stable strain-release radioiodination for thiol chemoselective bioconjugation. Bioconjug Chem. 2018; 29:467-472.

37. Feng B, Xu JJ, Bi YA, et al. Role of hepatic transporters in the disposition and hepatotoxicity of a HER2 tyrosine kinase inhibitor CP-724,714. Toxicol Sci. 2009; 108:492-500.

38. Doubrovin M, Kochetkova T, Santos E, et al. ${ }^{124} \mathrm{I}$-iodopyridopyrimidinone for PET of Abl kinase-expressing tumors in vivo. J Nucl Med. 2010;51:121-129.

39. Song H, Li Y, Lee J, Schwartz AL, Bu G. Low-density lipoprotein receptorrelated protein 1 promotes cancer cell migration and invasion by inducing the expression of matrix metalloproteinases 2 and 9. Cancer Res. 2009;69:879.

40. Su H, Seimbille Y, Ferl GZ, et al. Evaluation of $\left[{ }^{18} \mathrm{~F}\right]$ gefitinib as a molecular imaging probe for the assessment of the epidermal growth factor receptor status in malignant tumors. Eur J Nucl Med Mol Imaging. 2008;35:1089-1099. 